\section{SP1-67 CAN WE LIVE LONGER BY ELIMINATING DISEASES OF CIRCULATORY SYSTEM: AN ANALYSIS OF MCCD DATA IN SELECTED STATES OF INDIA}

doi:10.1136/jech.2011.142976n.44

${ }^{1}$ B K Gulati, ${ }^{2} \mathrm{M}$ Ubaidullah, ${ }^{1} \mathrm{~A}$ Pandey, ${ }^{1} \mathrm{D}$ Sahu. ${ }^{1}$ National Institute of Medical Statistics, ICMR, Ansari Nagar, New Delhi, India; ${ }^{2}$ S.V.University, Tirupati, Andhra Pradesh, India

Introduction Life expectancy is used as a development indicator for measuring developing of any country. Developed countries of the world have achieved considerable increase in life expectancy due to advancement in medical science. However, developing countries are still struggling hard to achieve the same level. The cause-specific death rates can demonstrate most prevalent diseases in the country. An age and cause specific death rate would give a more refined result of the same.

Methods Life table technique helps in examining the effect of elimination of a specific cause of death in life expectancy. One gets an idea about gain in life expectancy if one is able to control a specific disease. Diseases of circulatory system have become a major cause of death in developing countries including India. Therefore, present paper aims to construct such a life table using Medical Certification of Cause of Death data of Sample Registration System (SRS) in four selected states of India namely, Bihar, Rajasthan, Maharastra and Tamil Nadu to find out potential gain in average years of life due to prevention of mortality related circulatory system diseases.

Results The net gain in life expectancy at birth is found to be higher among females than males in all the states. Life tables are adjusted for the impact of differential risks of dying from other causes.

Conclusion This technique is useful in finding out potential benefits of cause elimination in health services and is essential for health policymakers and planners to set-up priorities in intervention programmes.

\section{SP1-68 EVALUATING COMMUNITY BURDEN OF DEPRESSION AND SUICIDAL TENDENCIES USING THE HOSPITAL DEPRESSION AND ANXIETY SCALE}

doi:10.1136/jech.2011.142976n.45

0 Popoola, ${ }^{*}$ E Owoaje. University College Hospital, Ibadan, Oyo state, Nigeria

Introduction Depression is a common mental health problem affecting human communities. A variety of screening tools have proved useful and this study explored the use of the Hospital Anxiety and Depression Scale among rural south western Nigerians. Methods Interviewer administered, community based survey of adults.

Results A total of 313 consenting community members were interviewed, $48.2 \%$ were female, sample mean age $34.25 \pm$ 14.82 years. Depression was established in $8.3 \%$ of individuals within the 2 week reference period (M:F sex specific prevalence of $10.5 \%$ and $6 \%$ respectively). Severe, moderate and mild depression was present in $46.1 \%, 23.1 \%$ and $30.8 \%$ of depressed individuals. Factors significantly associated with depression include recent bereavement OR 2.59 (95\% CI 1.07 to 6.25), somatic symptoms OR 2.77 (95\% CI 1.14 to 6.85 ) and accommodation problems OR $95 \%$ CI. Suicidal ideation and attempts were reported by $10.5 \%$ and $1.9 \%$ respectively. A multivariable logistic model which included variables significant at $10 \%$ retained only somatic symptoms as a predictor of depression in this study.

Conclusion The HADS is useful in community screening for depression. The observed high prevalence of depression despite a short reference period illustrates the importance of this condition in the study population. Health workers and local health services should be sensitised to consider a diagnosis of depression especially in the presence of unexplained somatic symptoms.

\section{SP1-69 BLO0D PRESSURE AND OBESITY IN EMIRATI CHILDREN AND ADOLESCENTS}

doi:10.1136/jech.2011.142976n.46

S Shah, ${ }^{*}$ F Al Maskari, A Al Mehairi, S Al Muhairi, M Al Naqbi, A Al Khouri. UAE University, AL AIN, United Arab Emirates

Objectives Although obesity is fast increasing, there are few data on the prevalence of high-normal or elevated blood pressure in United Arab Emirates.

Methods We conducted a school-based survey of a representative sample of youth $(n=1524)$ aged 12 to 18 years in Al Ain, UAE. BP measurements were made with a manual sphygmomanometer by trained nurses. Additional measures included height, weight, and abdominal circumference. BMI $\geq 85$ th and $\geq 95$ th percentiles were used to define overweight and obesity according to the 2000 CDC growth charts. The prevalence of "high normal" and "elevated" BP was assessed by comparing the subjects' SBP and DBP with age-, gender-, and height-specific 90th and 95th percentile reference values from the National High Blood Pressure Education Program.

Results A high proportion of Emirati children and adolescents (21\%) were obese compared to their non-local counterparts $(16 \%)$. Among study children $6 \%$ had elevated blood pressure. A high proportion $(19.1 \%)$ of obese children had elevated blood pressure compared to those who were overweight (4.8\%) and normal (2.1\%).

Conclusion The present findings emphasise the importance of the prevention of obesity in order to prevent future cardiovascular related problem such as hypertension.

\section{SP1-70 INADEQUATE CONTROL OF BLOOD PRESSURE IN HYPERTENSIVE PATIENTS ASSISTED BY A FAMILY DOCTOR PROGRAM}

doi:10.1136/jech.2011.142976n.47

${ }^{1} \mathrm{~B}$ da Silva Nalin de Souza, ${ }^{2} \mathrm{E}$ M Yokoo, ${ }^{2} \mathrm{M}$ Cagy, ${ }^{2} \mathrm{M} \mathrm{L}$ G Rosa. ${ }^{1}$ Sate University of Rio de Janeiro, Rio de Janeiro, Brazil; ${ }^{2}$ Fluminense Federal University, Niterói, Rio de Janeiro, Brazil

Introduction Hypertension is a major risk factor for cardiovascular disease. Despite the availability of various classes of anti-hypertensive medications, blood pressure (BP) control often remains poor. Lifestyle changes, if adopted, are effective at reducing BP. The aim of this study was to estimate the factors associated with inadequate BP control in hypertensive patients adherent to antihypertensive drug treatment assisted by a Brazilian Family Doctor Program (FDP).

Methods A cross-sectional study of men and women aged 20 years and over collected data on sociodemographic characteristics, comorbidities, lifestyle and food frequency, the latter using a questionnaire validated for the investigation of dietary habits. Blood and urine samples were obtained and anthropometric and nutritional studies performed.

Results Independent of time of treatment and systolic BP at admission to FDP, individuals with inadequate BP control had a higher body mass index (BMI) (Prevalence Ratio, PR 1.027 (95\% CI 1.009 to 1.045)), consumed more meat (PR 1.091 (1.022 to 1.165)), had higher levels of serum creatinine (PR 1.894 (1.241 to 2.892)) and more often had white skin colour (PR 1.363 (1.006 to 1.847)). 
Following adjustment for the sodium excretion index, an indirect measure of salt intake, there association with meat was no longer significant.

Conclusion Inadequate control of BP in patients who were being treated with anti-hypertensive drugs, even in a community assisted by a primary care program, was independently associated with modifiable factors including salt consumption and BMI. An association with skin colour and serum creatinine was also observed.

\section{SP1-71 HOW DOES THE AGE INFLUENCE ON THE RELATIONSHIP BETWEEN CENTRAL BLOOD PRESSURE AND CARDIOVASCULAR RISK FACTORS? CROSS-SECTIONAL ANALYSIS OF NAGAHAMA ZERO-JI PREVENTIVE COHORT PROJECT IN JAPAN}

doi:10.1136/jech.2011.142976n.48

${ }^{1} \mathrm{~S}$ Tokumoto, ${ }^{*}{ }^{1} \mathrm{Y}$ Takahashi, ${ }^{1} \mathrm{~T}$ Ishizaki, ${ }^{2} \mathrm{~K}$ MIYAKI, ${ }^{1} \mathrm{~T}$ Nakayama. ${ }^{1}$ Department of Health Informatics, Kyoto University School of Public Health, Kyoto City, Japan; ${ }^{2}$ National Center for Global Health and Medicine, Tokyo, Japan

Introduction Blood pressure is a well established cardiovascular risk factor. In addition to traditional blood pressure indexes such as SBP and DBP, estimation of central SBP (cSBP) has become available recently. The characteristics of cSBP have not been fully described. This study investigated the relationship between cSBP and known cardiovascular risk factors according to age group and comparing this with four traditional blood pressure indexes, SBP, DBP, PP and MBP.

Methods This study examined baseline cross-sectional data from 4375 healthy men and women aged $30-74$ years old who participated in a community-based cohort study conducted from 2008 to 2009. Linear regression analyses were carried out on the five blood pressure indexes adjusting for sex, age, BMI, triglycerides, HDLcholesterol, LDL-cholesterol, $\mathrm{HbA1c}$, state of anti-hypertensive medication, smoking, alcohol Intake, exercise, past history of cardiovascular diseases, ABI and cardio-ankle vascular index. We analysed the following two models: including these risk factors without sex and age (model 1); including age groups (age $<50$ / age $\geq 50$ ) (model 2). Subgroup analyses according to age group and sex were also performed.

Results In model 1 the $\mathrm{R}^{2}$ for sBP was 0.356 , and in model $2,0.385$. Incremental $\mathrm{R}^{2}$ was 0.028 between the models. Subgroup analyses showed five blood pressure indexes were explained by the total variances of known cardiovascular risk factors, more so for the younger than the older age group which was more apparent for cSBP than for the other blood pressure indexes.

Conclusion Known cardiovascular risk factors are associated with cSBP. This association is greater in younger $($ age $<50)$ than in older (age $\geq 50$ ) patients.

\section{SP1-72 FACTORS ASSOCIATED WITH DEPRESSION AMONG ELDERLY IN KARACHI PAKISTAN: A MATCHED CASE CONTROL STUDY}

doi:10.1136/jech.2011.142976n.49

${ }^{1} \mathrm{~K}$ Nanji, ${ }^{2} \mathrm{M}$ Kadir, ${ }^{3} \mathrm{~K}$ Ahmed, ${ }^{4} \mathrm{H}$ Naqvi. ${ }^{1}$ Department of Family Medicine, The Aga Khan University, Karachi, Pakistan; ${ }^{2}$ Department of Community Health Sciences, The Aga Khan University, Karachi, Pakistan; ${ }^{3}$ Department of Opthalmology, The Aga Khan University, Karachi, Pakistan; ${ }^{4}$ Department of Psychiatry, The Aga Khan University, Karachi, Pakistan

Introduction The increase in life expectancy due to epidemiologic transition has brought about increased numbers of certain mental illnesses, namely depression. There is scarcity of analytical base epidemiological studies on this issue. This study was conducted to identify factors associated with depression among elderly $(>60)$ in Karachi, Pakistan.

Method A multicentre matched case control study was conducted. A total of 234 depressed cases and 468 non-depressed controls (1case:2controls) were selected from four tertiary care hospitals in Karachi. Cases were recruited from psychiatric OPD whereas; controls were recruited from other OPD's (except psychiatric) and were individually matched on age and gender with cases. Ascertainment of cases and controls was done by psychiatrist and Geriatric Depression Scale. There was substantial agreement between the psychiatrists for diagnosing depression ( $\kappa$ : 0.66). A pilot tested structured questionnaire was administered and analysis was performed through conditional logistic regression using SPSS 11.5

Results Risk factors for depression were, living in nuclear family system, (mOR: 4, CI 2.1 to 4.9), self reported difficult situation during past 1 year (mOR: 9, CI 6.0 to 19.7), hearing and/or visual impairment (mOR: 5, CI 2.0 to 9.8), presence of $>2$ NCD (mOR: 2 , CI 1.0 to 3.4). However, interacting with friends or relatives had a protective effect against depression (mOR adj: $0.3,95 \%$ CI 0.3 to 0.7$)$.

Conclusion Interventions should be taken to reduce modifiable factors and non-modifiable factors should be used to identify high risk group for primary prevention. However factors identified through this study may vary in its association with depression across different communities.

\section{SP1-73 THE PREVALENCE OF COGNITIVE IMPAIRMENT AND ITS ASSOCIATION WITH SOCIAL SUPPORT IN ELDERLY PEOPLE IN KARACHI, PAKISTAN}

doi:10.1136/jech.2011.142976n.50

${ }^{1} \mathrm{M}$ Bhamani, ${ }^{1} \mathrm{~K}$ Nanji.* ${ }^{1}$ Department of Community Health Sciences-The Aga Khan University, Karachi, Pakistan; ${ }^{2}$ Department of Family Medicine-The Aga Khan University, Karachi, Pakistan

Introduction Cognitive impairment is one of the commonest mental health problems in the elderly. The objective of this study was to estimate the prevalence of cognitive impairment in the elderly (age $>60$ years) population in Karachi, Pakistan and to determine the association between social support and cognitive impairment in this group.

Methods A population based cross sectional study of the elderly in Karachi Pakistan was carried out between November 2010 and March 2011. Participants were randomly recruited in 30 clusters from 18 towns in Karachi using a multi-stage cluster sampling technique. Face-to-Face interviews were conducted with 730 eligible participants using structured questionnaires. The prevalence of cognitive impairment was assessed through Mini Mental State Examination (MMSE). For illiterate participants cognitive impairment was defined as a score of $<21$; for literate $<23$. Data analysis is underway using SPSS 16. Logistic regression analysis will be used to determine the association between social support and cognitive impairment.

Results This study is currently in progress. We anticipate that prevalence of cognitive impairment will be higher and will be attributable to the social support received by the elderly.

Conclusion Conclusion will be based on the results of this study. However, we suggest that the younger generation should be sensitised towards their responsibilities for the elderly and the government should also formulate effective health policies for this group. Further, longitudinal studies should be planned to identify other factors that are associated with cognitive impairment in the elderly. 